\title{
Eu, meu lugar e eu mesmo: explorando a ligação entre consumidores e lugares com um tempero do Nordeste brasileiro
}

\author{
VITOR MOURA LIMA ${ }^{1}$ \\ Rafael Cuba Mancebo ${ }^{2}$ \\ Luís Alexandre Grubits de PaUla Pessôa ${ }^{2}$ \\ ALESSANDRA DE SÁ MELLO dA COSTA ${ }^{2}$ \\ 1 YORK UNIVERSITY / SCHULICH SCHOOL OF BUSINESS, TORONTO - ON, CANADÁ \\ 2 PontifícIA UnIVERSIDAde CATÓLICA do RIO DE JANEIRO (PUC-RIO), RIO DE JANEIRO - RJ, BRASIL
}

\section{Resumo}

Os consumidores, mais ou menos conscientemente, conectam sua identidade a lugares para dar sentido às suas vidas. Nesta pesquisa, discutimos o processo pelo qual os consumidores fazem a ligação entre sua identidade a ambientes comerciais, baseando-se na teoria do Extended Self e perspectivas do Place Attachment. Por meio de observações, entrevistas em profundidade e análise de discurso, este estudo explora esse vínculo, tendo como contexto de pesquisa uma feira regional que funciona como um centro cultural de tradições do nordeste brasileiro. Os achados sugerem que o vínculo entre consumidores e lugares comerciais ocorrem de diferentes formas, com base não apenas em suas narrativas pessoais, mas também pela espacialidade física do local.

Palavras-chave: Extended Self. Place Attachment. Comércio. Semiótica. Brasil.

\section{Me, my place, and I: exploring consumer-place attachment in Brazil's Northeast region}

\section{Abstract}

Consumers, whether more or less consciously, attach their identity to places in order to give meaning to their lives. In this research, we discuss the process by which consumers attach their identity to commercial settings, based on the extended self and place attachment theories. Through observations, in-depth interviews, and discourse analysis, this study explores the bonds people make with a place, taking a Brazilian heritage market as a research context. The findings suggest that the link between consumers' identities and commercial settings occurs in different forms, based not only on their self-narratives but also on the physical space.

Keywords: Extended Self. Place Attachment. Commercial Settings. Semiotics. Brazil.

Yo, mi lugar y yo mismo: explorando la conexión entre los consumidores y los lugares con "sabor" de la región noreste de Brasil

\section{Resumen}

Nuestra existencia como seres humanos está directamente vinculada a lugares donde se vive una experiencia particular. Los consumidores conectan más o menos conscientemente su identidad a lugares para dar sentido a sus vidas. En esta investigación, discutimos el proceso mediante el cual los consumidores vinculan sus identidades a los entornos comerciales, con base en la teoría del yo extendido y las perspectivas del place attachment. A través de observaciones, entrevistas en profundidad y análisis del discurso, este estudio explora este vínculo. El contexto de la investigación fue una feria regional que actúa como un centro cultural de las tradiciones del noreste de Brasil. Los resultados sugieren que el vínculo entre los consumidores y los lugares comerciales se produce de diferentes maneras, no solo en función de sus narraciones personales, sino también de la espacialidad física del lugar.

Palabras clave: Yo extendido. Place attachment. Comercio. Semiótica. Brasil. 


\section{INTRODUÇÃO}

Uma parte de nós está diretamente relacionada ao lugar em que nascemos, onde escolhemos morar, e aos lugares que frequentamos, dado que cada um deles é composto por um conjunto de significados e valores que são articulados em nossas narrativas pessoais (GAVIRIA, CARDOSO, SCARABOTO et al., 2019). Nesses locais, com maior ou menor influência, os objetos, assim como as pessoas e suas histórias, contribuem para a representação dos mais variados tipos de projetos de identidade. Portanto, quando dizemos "sou deste lugar" ou "esse lugar é o meu lugar", além de indicar que pertencemos a algum lugar, indicamos que o lugar também nos pertence. Em outras palavras, somos parte do lugar, assim como o lugar é de alguma forma parte de nós. À luz dessa abordagem e de acordo com James (1890), um lugar pode ser visto como uma posse diretamente ligado à identidade de alguém.

Ao longo dos anos, diferentes abordagens foram utilizadas para explorar a relação entre consumidores e suas posses (e.g., BRAGA e SUAREZ, 2018). Considerando a teoria do Extended Self (BELK, 1988), seus contrapontos (e.g., AHUVIA, 2005) e seu desenvolvimento (e.g., BELK, 2015, 2013; RUVIO e BELK, 2018), a aura ou a magia de um lugar podem nos contaminar e serem contaminadas por nós, bem como, nos fazer criar laços significativos com uma casa (BORRAZ, 2019), uma cidade ou mesmo um ambiente comercial, como no caso de Holbrook (1998) e seu amor por uma loja de jazz. Independentemente de sua contribuição seminal, Belk (1988) apresenta brevemente o lugar, comercial ou não, como uma forma de posse e como parte da relação com a construção da identidade dos indivíduos.

Apesar de muitas pesquisas em estudos do consumo terem demonstrado que experiências significativas podem ser fornecidas por gestores que tenham o objetivo de estabelecer laços emocionais entre os negócios e seus clientes, o processo pelo qual os consumidores associam sua identidade a esses ambientes comerciais foi amplamente negligenciado. Embora estes estudos contribuam para a nossa compreensão sobre o papel dos locais de serviços, por exemplo, no despertar de emoções e significados, tem sido dada pouca atenção à forma como isso acontece (EROGLU e MICHEL, 2018; DEBENEDETTI, OPPEWAL e ARSEL, 2014). Neste cenário, há uma oportunidade para pesquisas que considerem o processo de conexão entre consumidores e os espaços físicos comerciais, e as transformações que ocorrem nas suas autonarrativas, como sugerido por Kreuzer, Mühlbacher e von Wallpach (2018), Seamon (2018), e Debenedetti, Oppewal e Arsel (2014). Assim, este estudo é orientado pela seguinte questão de investigação: de que maneira os consumidores associam suas identidades aos locais comerciais?

Uma melhor compreensão do vínculo entre as identidades dos consumidores e as configurações comerciais é importante por razões teóricas e práticas. Primeiro, esse conhecimento pode contribuir para os esforços de pesquisa focados no processo pelo qual os consumidores vinculam suas identidades aos locais comerciais (DEBENEDETTI, OPPEWAL e ARSEL, 2014). Segundo, como uma contribuição gerencial, já que nosso foco está nos significados contextuais e na experiência vivida com a qual este estudo também pode ajudar os gerentes no desenvolvimento de estratégias de experiência de marca (PULH, MENCARELLI e CHANEY, 2019).

Nossos argumentos se baseiam na teoria do Extended Self (BELK, 1988) e das perspectivas do Place Attachment (SEAMON, 2018; ALTMAN e LOW, 1992). Em relação à coleta de dados, utilizamos múltiplas fontes, como observações e entrevistas (BELK, FISCHER e KOZINETS, 2013), que foram analisadas a partir da semiótica discursiva da linha francesa (GREIMAS e COURTÉS, 1983). Essas abordagens são adequadas para este estudo principalmente devido ao uso da Trajetória Generativa de Significado (GREIMAS e COURTÉS, 1983) e da Axiologia do Consumo (FLOCH, 1988) como marcos analíticos, uma vez que permitem ao pesquisador observar aspectos concretos e sensíveis do fenômeno.

Como contexto de pesquisa, foi escolhida a Feira de São Cristóvão, que é um mercado localizado no Rio de Janeiro, e considerado patrimônio imaterial do Brasil. As razões para esse contexto são: (1) é um ambiente físico comercial especializado em uma variedade de serviços e produtos nordestinos; (2) é uma representação da tradicional cultura nordestina brasileira no sudeste do país; (3) o local é considerado "lar" dos imigrantes nordestinos brasileiros (JÚNIOR e PORTO, 2002). Para apresentar a pesquisa, este artigo está organizado da seguinte forma: Primeiro, o esforço de revisar a vasta literatura e estruturar os argumentos com base nas formas de conexão consumidor-lugar, para em seguida apresentar os métodos de pesquisa. Na seção seguinte são apresentados os resultados, seguidos da discussão teórica e as implicações gerenciais. A parte final deste estudo aborda as limitações e sugestões para estudos futuros. 


\section{REVISÃO DE LITERATURA}

\section{Identidade e a teoria do Extended Self}

A identidade do consumidor não pode ser vista como estável ou fixa, dado que há no contexto diferentes possibilidades de representações que competem sobre quem podemos ou devemos ser. Em vez disso, a identidade deve ser considerada como um projeto em andamento, uma narrativa interminável, um estado permanente de devir (SCHAU, 2018; ZANETTE, LOURENÇO e BRITO, 2013; RUVIO e BELK, 2018). Dada essa natureza, a identidade é criada e recriada, construída e desconstruída durante nossas interações sociais diárias. Nesse contexto, diferentes abordagens investigaram o papel do consumo na criação de identidades, nas quais o princípio central é que o ato de consumir é uma chave para permitir uma pluralidade de identidades, pois somos o que consumimos e consumimos para comunicar quem somos. (ABDALLA e ZAMBALDI, 2016; BELK, 2013, 1992, 1988).

A cultura de consumo contemporânea é vista como um local onde os consumidores, individualmente ou coletivamente, podem encontrar a produção e circulação de significados, bem como representações discursivas para seus projetos de identidade desejados (FERREIRA e SCARABOTO, 2016). Considerando bens de consumo, serviços, informações ou mesmo experiências, os significados não estão apenas inscritos em seu uso, mas também em seu aspecto simbólico de um determinado contexto (MARCOUX, 2017). Nesse sentido, o que compramos ou recebemos, ou seja, nossos bens, tem o poder de agir como extensões de nós mesmos, se tornando fundamentais para nossas vidas.

A teoria do Extended Self, (BELK, 2015, 2013, 1988) propõe que nosso apego a lugares, objetos, partes do corpo, para o bem ou para o mal, é essencial para definir a nossa identidade. Aqui, o "meu" é adicionado ao "eu". Conforme destacado por Belk (1988), nossa capacidade de dominar um objeto (por exemplo, dominar a escultura), o ato de criá-lo (por exemplo, comprar um produto) e conhecê-lo (por exemplo, o desejo por ele) são maneiras pelas quais as posses podem tornar-se parte do Eu (Self) e estruturar as identidades.

Por exemplo, o lugar mais evidente que pode ser anexado ao Eu provavelmente é aquele em que vivemos ou moramos: nossa casa (GOWRICHARN, 2019). É possível deduzir que ele pode ser visto como o foco de vínculos emocionais, atividades de lazer e de responsabilidades financeiras (ROSENBERG, 2012). Ele tem o poder de moldar, definir e nos guiar na busca pelo nosso lugar no mundo (GIOVANARDI e LUCARELLI, 2018). Um lugar, como a nossa casa, é um local de autoexpressão (BELK, 1988), e pode ser tão importante quanto os objetos no processo de representação de identidades (ISABELLE, DOMINIQUE e STATIA, 2019). Assim, as posses ligadas ao Eu têm o poder de promover e comunicar diferentes níveis de identidade (ou seja, como indivíduo, família, comunidade e grupo), que são fundamentais para a nossa existência em uma determinada realidade social (BELK, 2013, 1988; THOMPSON e TAMBYAH, 1999).

\section{Perspectivas do Place Attachment}

Locais de nascimento, casamento, formatura, morte e outros são definitivamente parte de nossa história e identidade. Devido à sua natureza, um lugar pode provocar emoções intensas, memórias e vários tipos de comportamento (CANNIFORD, RIACH e HILL, 2018). Em outras palavras, nosso senso próprio poderia estar diretamente ligado a lugares geográficos em que ocorrem rituais que geram significados, uma vez que o vínculo com eles compartilha a mesma fenomenologia que o vínculo com nosso corpo e outros objetos (CASEY, 2009; BELK, 1992).

A partir de uma perspectiva fenomenológica, um lugar pode ser entendido como qualquer lócus ambiental no qual uma pessoa, ao mesmo tempo, age, experimenta uma dada realidade e concebe significado espacialmente (LIMA et al., 2019; CHATZIDAKIS, MCEACHERN e WARNABY, 2014). Uma cidade, uma casa, um brechó, por exemplo, não podem ser vistos como separadas da pessoa a elas associadas; ao contrário, deve ser visto como um fenômeno pessoa-experiência-lugar (SEAMON, 2018). Assim, o significado concebido e articulado nessas experiências é incorporado à identidade e às narrativas dos consumidores, tornando o local parte de suas posses (BELK, 1992, 1988).

Pesquisadores de consumo têm investigado conexões com lugares a partir de várias perspectivas (GIOVANARDI e LUCARELLI, 2018). Por um lado, há uma dimensão sobre a relação com espaços privados, como uma casa. Nesse caso, como exemplo, Hirschman, Ruvio e Belk (2012) estudaram garagens americanas para entender os papéis dos espaços liminares no gerenciamento de posses, seus significados e sua importância para a identidade. Outro exemplo dessa abordagem é o estudo de Mehta e Belk (1991), que observou bens e acessórios de índios residentes na Índia e nos EUA, para entender como eles articulam 
e protegem sua identidade. Por outro lado, uma perspectiva diferente sobre a importância das dimensões geográficas na pesquisa de marketing é fundamentada por uma visão mais estrutural e ampla de sua constituição. Por exemplo, Castilhos, Dolbec e Veresiu (2017) apresentaram uma abordagem geográfica para observar a criação da dinâmica do sistema de mercado. Dados os fatores incontroláveis decorrentes dessa dinâmica, os consumidores encenam seus projetos de identidade sem estarem conscientes de suas práticas. Conforme destacado por Veresiu e Giesler (2018), há casos em que fatores institucionais, como desequilíbrios de poder neocolonial entre culturas colonizadas (envio de imigrantes) e colonizadoras (recebimento de imigrantes), desempenham um papel fundamental na dinâmica do vínculo consumidor-local.

O processo de extensão do self a partir dos lugares e torná-los parte de nossos bens, seja com uma experiência individual ou coletiva do lugar, pode compartilhar o mesmo princípio da visita a locais religiosos, à medida que tentamos incorporar suas características mágicas e aspectos simbólicos, na tentativa de alcançar um certo nível de transcendência e encontrar novas camadas de nós mesmos (TERZIDOU, SCARLES e SAUNDERS, 2017). Tendo lugares como posses e observando nosso apego a eles, é possível definir quem somos, as narrativas de nossas vidas e o futuro que se aproxima.

\section{CONTEXTO E MÉTODOS}

\section{Contexto de pesquisa}

A Feira de São Cristóvão (FSC) completou 75 anos de existência em 2019. A Feira começou na década de 1940, após a construção da rodovia Rio-Bahia, funcionando como um ponto de encontro de imigrantes do Nordeste do Brasil, que chegavam ao Rio de Janeiro em busca por melhores condições de vida. Essa área se tornou o local de chegada e ponto de encontro dos nordestinos, não sendo apenas um lugar para se divertir, mas também onde podiam trocar produtos e mercadorias típicos de suas regiões. Além de suas características comerciais, desde 2010 a FSC é Patrimônio Cultural Imaterial do Brasil. Em termos de seleção do FSC como contexto de pesquisa, nossa justificativa é sustentada pelo fato de ser um ambiente físico comercial especializado em cultura brasileira, com produtos, serviços e experiências culturais do Nordeste. Além disso, o local é considerado o "lar" dos imigrantes nordestinos brasileiros distantes de sua região de origem (JÚNIOR e PORTO, 2002).

\section{Coleta de dados}

A coleta de dados foi iniciada em 2013 como parte de um projeto de pesquisa longitudinal que observou aspectos relacionados a diáspora, história e as práticas de consumo. Com o objetivo de desvelar discursos ideológicos baseados na mídia, a primeira etapa durou até 2015 (MANCEBO, 2016). Ao longo desses dois primeiros anos, coletamos dados de arquivo, tivemos conversas informais e nos envolvemos na observação participante (BELK, FISCHER e KOZINETS, 2013). De 2015 a 2017, o interesse mudou para os discursos institucionais, possibilitando a comparação e o contraste de diferentes perspectivas discursivas sobre o mesmo objeto focal. Nesse caso, usando uma abordagem netnográfica (KOZINETS, 2019), os dados foram recuperados dos canais de comunicação oficiais da FSC, como a página do Facebook. Semelhante a estudos anteriores (e.g., KOZINETS, PATTERSON e ASHMAN, 2017), as abordagens exploratórias e (n)etnográficas ajudaram no delineamento das entrevistas e insights. Observar de perto as atividades cotidianas da FSC, enquanto ouvia histórias, conhecia visitantes, proprietários de barracas e alguns de seus gerentes, desempenhou um papel crítico na compreensão do processo de vinculação e criação de significado dos consumidores e seus vínculos ao local.

De 2017 a 2019, dadas as visitas contínuas do segundo autor ao mercado, tornou-se claro o interesse de diferentes perfis de consumidores. Durante as interações sociais, as narrativas, propósitos e significados da FSC para os nordestinos ressoavam diferentemente daqueles nascidos em outras regiões brasileiras. Esses achados, de alguma forma, delinearam a terceira etapa do nosso projeto de pesquisa longitudinal, que é o presente estudo. Portanto, o foco foi reduzido para explorar a conexão consumidor-lugar, considerando apenas os nascidos no nordeste brasileiro e atualmente morando longe da terra natal. Os critérios de seleção para os entrevistados foram baseados em seleção teórica (MILES e HUBERMAN, 2014). Como mantivemos um registro de nossos entrevistados (e.g., nome, idade, cidade natal, residência atual, o objetivo da visita, e-mail e número de telefone), entramos em contato com eles e realizamos 15 entrevistas em profundidade em maio 2019. As entrevistas variaram de 30 a 70 minutos, em média, e começaram com uma breve apresentação da investigação, seguida de uma grande pergunta sobre a ida a feira, na qual eles foram convidados a falar sobre experiências no local (BELK, FISCHER e KOZINETS, 2013). 


\section{Análise de dados}

Tendo em vista a abordagem epistemológica e metodológica baseada no discurso que o projeto de pesquisa como um todo tem, nosso conjunto de entrevistas foi analisado seguindo a Semiótica Discursiva francesa (GREIMAS e COURTÉS, 1983). Essa abordagem semiótica compreende os aspectos inteligíveis e sensíveis do fenômeno (PESSÔA, COSTA, MELLO et al., 2020; LIMA e PESSÔA, 2018; FLOCH, 1988). Para tanto, foi utilizada uma adaptação do modelo de Percurso Generativa de Significação (PGS), considerando suas três partes: (1) nível fundamental; (2) nível narrativo; e (3) nível discursivo. Conforme previsto no nível inicial de análise, o Fundamental, nosso primeiro passo foi observar os valores de oposição primários (por exemplo, natureza e cultura) que emergiram de nosso conjunto de dados. Dados os resultados da codificação inicial, decidimos combinar nossa estrutura analítica com a Axiologia do Consumo de Floch (1988), o que nos ajudou a alcançar uma melhor compreensão do fenômeno. Com base no quadrado semiótico, Floch (1988) designa valores atribuídos aos espaços físicos nas seguintes categorias: Prático, Utópico, Crítico e Lúdico. Em seguida, no nível narrativo, demos atenção a vários elementos das experiências vividas pelos consumidores, vistos como narrativas de consumo. Nesta fase do PGS, o processo de como os consumidores vinculam suas identidades a locais comerciais começou a ficar mais claro, uma vez que foi possível desdobrar no esquema de narrativa canônica (ENC) (e.g., qual objeto permitiu que alguém fizesse alguma coisa). Finalmente, para o nível discursivo, identificamos as noções dos entrevistados sobre os papéis temáticos da temporalidade e espacialidade na FSC, e os valores previamente identificados que promovem diferentes formas de identidades. Por uma questão de confiabilidade desta pesquisa, realizamos várias rodadas de discussão (WALLENDORF e BELK, 1989). As reflexões individuais sobre os dados promoveram insights sobre o fenômeno e foram cruzadas por um processo coletivo de triangulação para comparar classificação, padrões e coerência com o que foi proposto.

\section{Resultados}

O processo pelo qual os consumidores vinculam suas identidades a estabelecimentos comerciais pode ser visto por uma abordagem tríplice, seguindo o modelo PGS (GREIMAS e COURTÉS, 1983). Primeiro, as experiências pessoais dos consumidores foram organizadas na Axiologia do Consumo de Floch (1988), dados os valores estruturantes identificados (ou seja, Nível Fundamental). Segundo, desenvolvemos um padrão em termos de experiências do consumidor que foram estruturadas no ENC (isto é, nível narrativo). Terceiro, observamos que as experiências e a representação de identidades também têm uma certa conexão nas áreas físicas do FSC (ou seja, nível discursivo). Esses relacionamentos também foram organizados com base na proposta de Floch (1988) de conectar cada um dos níveis do PGS a diferentes perfis de consumidores.

\section{Nível Fundamental: A axiologia do consumo e a conexão consumidor-lugar}

Dado o uso do PGS (GREIMAS e COURTÉS, 1983), é essencial apresentar o conceito do Nível Fundamental, pois ele fornece os valores que funcionam como pilares para uma determinada experiência no mundo, ou como neste caso, um projeto de identidade. O nível fundamental é o mais abstrato do PGS. Como Floch (1988) constatou em sua pesquisa, hipermercados, mercados e feiras podem ter consumidores com valores mais instrumentais de consumo. Valores práticos, portanto, entendidos como valores utilitários. Na FSC, por exemplo, esses valores estão relacionados a estacionamento, segurança, diferentes tipos de alimentos e ervas e especiarias que não podem ser comprados em um supermercado comum. Nessa perspectiva, a FSC é vista como um lugar para adquirir itens essenciais para as experiências pessoais dos consumidores, sustentados pelo ato de criar (por exemplo, compras) de acordo com a teoria do Extended Self (BELK, 1988). A declaração a seguir ilustra a situação.

Entrevistado: "Temos que vir duas vezes por mês aqui para São Cristóvão e por isso chegamos aqui, passamos um tempinho, observamos algumas coisas, almoçamos, compramos alguns produtos do Nordeste, como pimenta e farinha de mandioca. Isso se comprarmos alguma coisa, senão só saímos e almoçamos."

Embora esses valores sejam fundamentados na instrumentalidade, eles são fundamentais para a conexão emocional entre o consumidor e o local comercial. Portanto, o vínculo também estaria relacionado à possibilidade de não encontrar o item ou serviço desejado. Como destacaram Belk (1988) e Guillard (2017), ser assaltado, perder e / ou não encontrar uma posse é, de alguma forma, sentido como uma perda de parte da autonarrativa do consumidor. Portanto, sem a possibilidade de acessar e comprar bens e serviços regionais, o consumidor tende a ter um impacto negativo sobre o lugar e a memória emocional (RISHBETH e POWELL, 2013). O trecho a seguir ilustra o aspecto da ausência. 
Entrevistado: "Um mercado no Rio de Janeiro para produtos nordestinos e para as pessoas que vêm à noite ... você tem músicas e shows ... você tem essa opção de varejo adicional para o Rio à noite. É seguro e você certamente encontrará o que precisa a partir daí. Não sei o que faria se o mercado não estivesse aqui. Eu amo o lugar."

Por outro lado, os valores utópicos são entendidos como valores existenciais. Floch (1988) enfatiza que o termo utópico é usado como "o objetivo final", ou seja, é quando os consumidores encontram seus valores fundamentais. Conforme apontado por vários entrevistados, estar apegado ao local está diretamente relacionado à noção de "estar no mundo deles". Em alguns casos, depois de anos longe do nordeste brasileiro, muitas pessoas encontram uma referência para sua identidade na FSC, que pode ser vista como um local que fornece alguma segurança ontológica para suas autonarrativas (GAVIRIA, CARDOSO, SCARABOTO et al., 2019). A declaração a seguir ilustra o caso.

Entrevistado: “tem a lembrança nordestina, que é a tradição regional 'dentro' do Sul... E a gente não morreu, a origem tá aqui presente, a recordação da terrinha... a gente chama de 'nosso quintal', 'nossa segunda casa". Minha cidade é aconchegante ... todo mundo conhece todo mundo. Isso me leva de volta anos atrás. Às vezes, me lembro da minha infância e do quanto estava feliz. Eu gostaria que toda a minha família estivesse aqui agora."

De fato, muitas das experiências relatadas são marcadas pela busca de algum contato com os aspectos culturais do Nordeste, que serve como uma maneira de matar a saudade da terra natal, destacando o ato de conhecer a posse (e.g., desejar), como proposto por Belk (1988). Nesse cenário, esse tipo de apego ao local do consumidor pode ser visto como o elo que as pessoas têm com a identidade histórica de um determinado local, comunidade ou até mesmo objetos (LOW, 1992). A FSC é vista principalmente como um local para matar a saudade das cidades de origem dos consumidores, não apenas em termos de produtos que você pode comprar, mas também devido à estrutura física do local, que pode ser vista como parte de seu senso de lar (MCCRACKEN, 1989). A frase a seguir destaca esse aspecto.

Entrevistado: “Esses becos, as ruas, têm os nomes dos 'bairros' lá do Nordeste... Campina Grande, João Pessoa e assim vai... Toda parte do Nordeste tem o nome aqui e representa o Nordeste e a gente."

A terceira categoria de Floch (1988), Valores críticos, nega os valores utópicos, mas complementa os valores práticos. Nesse caso, é a ideia de custo / benefício que permeia os depoimentos dos visitantes da FSC e que vão comprar algum utensílio específico a um preço mais baixo ou até mesmo procurar um produto regional de melhor qualidade. Ou seja, a experiência vivida de estar no mercado e consumir é o que sustenta um certo tipo de vínculo consumidor-lugar, já que dominar uma posse, como conhecer o lugar certo para estar e comprar, é uma das dimensões da teoria do Extended Self (BELK, 1988). O trecho a seguir ilustra valores críticos.

Entrevistado: "Você sai daqui já com um tomate, uma cebola, um monte de coisa. Coisas típicas, quem gosta, camarão seco, com preços muito bons, tem um supermercado aqui dentro. Você não encontra produtos baratos como esses lá fora. Se você for a um grande supermercado, gastará todo o seu salário lá. Não tenho dinheiro para gastar ... você sabe. Aqui posso ter tudo o que quero por um preço razoável e sei que é original. Tem outro lugar no centro do Rio que vende produtos da minha cidade, mas não confio neles. Aqui é o lugar."

Os valores lúdicos são a negação dos valores práticos e, como sugere Floch (1988), são guiados ao prazer e à diversão. Vários entrevistados afirmaram ter vínculo com o local, dado o hábito de ir para lá para ser quem eles querem ser e mergulhar em uma experiência única. Conforme observado por Cova, Carù e Cayla (2018), essas experiências, mais ou menos escapistas, são fundamentais para o processo de construção da identidade. Para apoiar esse argumento, a declaração do próximo entrevistado apresenta esses valores.

Entrevistado: “O clima do Nordeste é esse; as pessoas a vontade, ninguém te julgando e aí você pode cantar, comer e participar do Karaokê sem nenhum problema. Eu sei dançar meu próprio estilo ... danço muito o Forró e não ligo. Chega uma hora da noite que tiro os sapatos e continuo dançando até que alguém me chute para fora do lugar. Gosto de beber minha cerveja, às vezes fico um pouco alegrinha ... você entende o que quero dizer. Tem muito amor aqui. As pessoas se mudaram de cidade e depois querem ser felizes. Venha ... pare de me fazer perguntas. Dança Comigo..." 
Esse "sentimento de descontração" expresso pela maioria dos entrevistados é o que permite a realização de "atividades gratuitas por excelência" na FSC, e que define essencialmente a avaliação lúdica. Assim, o apego consumidor-lugar está alinhado com o consumo de experiências para encontrar sentimentos, fantasia e diversão, às vezes buscando escapar de sua realidade (HIRSCHMAN e HOLBROOK, 1982) e "ir para outro lugar". Nesse ponto, é possível articular todos os quatro tipos de valores em um quadrado semiótico, como proposto por Floch (1988). A Figura 1 ilustra isso.

Figura 1

Axiologia da conexão consumidor-lugar

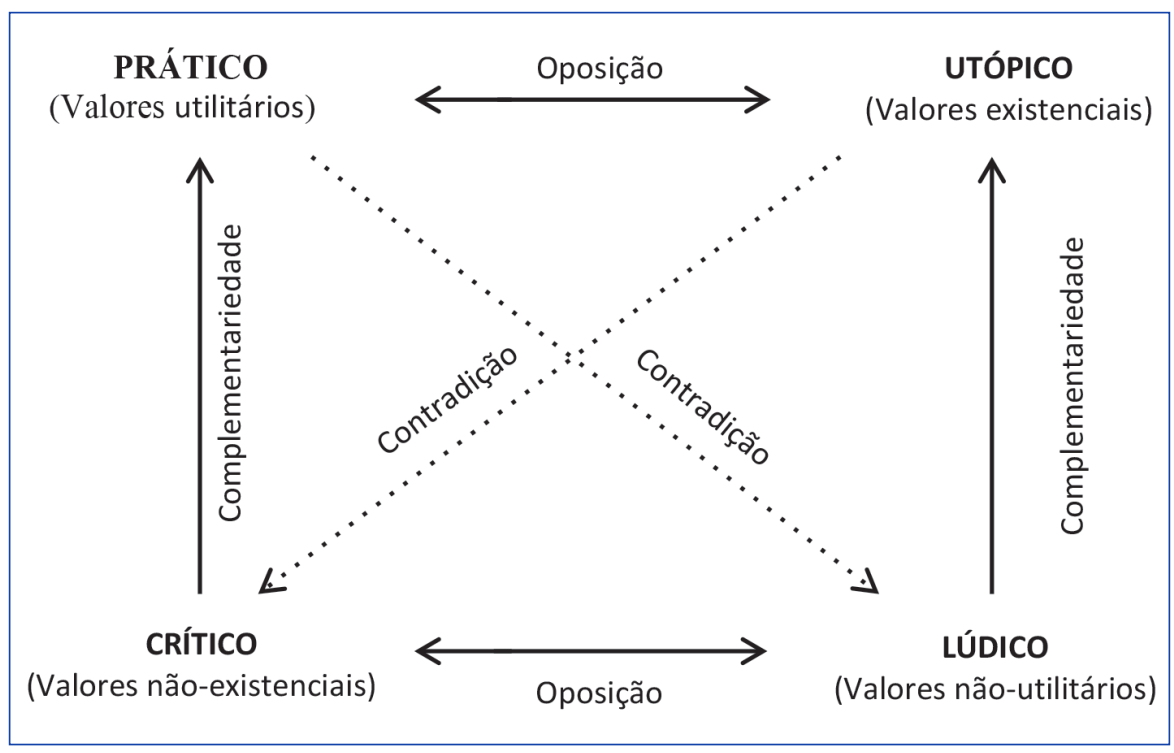

Fonte: Floch (1988).

Embora os valores utópicos prevaleçam mais nas experiências dos consumidores enquanto se vinculam ao ambiente comercial, vale ressaltar que cada tipo de valor desempenha um papel importante para cada um dos entrevistados no processo de extensão do self.

\section{Nível narrativo: padrão narrativo para o consumer-place attachment}

Com base na axiologia concebida, avançamos para o desenvolvimento da ENC, que é composta por regimes de doings e beings a partir de quatro estágios: (1) Manipulação; (2) competência; (3) desempenho; e (4) sanção (GREIMAS e COURTÉS, 1983). Considerando a experiência vivida de consumo de cada entrevistado como um conjunto de narrativas, foi possível organizar seus aspectos e valores comuns identificados no nível fundamental em uma narrativa padrão. Ou seja, o Nível Narrativo forneceu um entendimento das manifestações de valores nas representações de identidade dos consumidores.

$\mathrm{Na}$ fase de Manipulação, os indivíduos são influenciados pelas narrativas de querer fazer e/ou ter que fazer, que podem se materializar na forma de contratos e são vistas em declarações sobre a expectativa de encontrar determinados bens, serviços, e experiências no local. Assim, entende-se que, a partir da pesquisa, a FSC foi construída com o desejo de ser o Nordeste no Rio de Janeiro com todos os seus significados culturais e como um local onde os visitantes esperam encontrar não apenas produtos nordestinos, mas também simbolismo regional. Como destacou Belk (1988), conhecer e, neste caso, desejar a FSC e o que ela oferece, é uma das formas de ligação emocional e simbólica com o lugar, e a transformação resultante do lugar como parte do self.

Durante a etapa de Competência, a segunda fase, há uma transformação feita pelo consumidor, que pode realizar uma determinada ação a partir do saber fazer e /ou ser capaz de fazer. Esta discussão está relacionada ao conhecimento sobre ofertas que permitem aos visitantes interagir e se beneficiar da experiência nordestina. Os entrevistados demonstraram familiaridade com a planta da Feira, de forma a encontrar diferentes atividades, que variam de eventos musicais, venda de 
ervas, especiarias e artesanato à restaurantes típicos. Durante as visitas, a experiência de consumir alimentos confeccionados por especialistas e nativos nordestinos intensifica a importância do ambiente comercial para o self. Esse aspecto está alinhado com a terceira fase da ENC, que é o desempenho. Aqui, realmente ocorrem transformações, ou seja, uma passagem de um estado para outro a partir de um conjunto de práticas, como almoçar. Como mostra Tsai (2016), o ato de provar a culinária local fornece um meio crucial para representações e transferência da cultura de um determinado destino, proporcionando experiências inesquecíveis. Além disso, os sons e estilos musicais da região, que variam do Forró tradicional às versões mais modernas do ritmo, também são fundamentais para a ligação com o local e os processos de construção de identidade. Consumir produtos locais, participar de apresentações e ouvir histórias sobre sua terra natal e o mercado, ou seja, ter contato com a cultura nordestina é o que permite que os consumidores sejam positivamente sancionados, que é a fase final da ENC. A etapa da Sanção está relacionada à constatação de que o desempenho foi cumprido, o que, consequentemente, promove o reconhecimento do consumidor. No caso da ENC, pode ser visto como uma interação positiva entre o que é encontrado no mercado e as expectativas do consumidor visitante. Esse aspecto lança luz sobre o ato de criar (e.g., comprar) como uma forma de estender o self a uma posse (BELK, 1992, 1988), que, neste caso, é a FSC. A Figura 2 exemplifica a ENC para a conexão consumidor-lugar.

Figura 2

Esquema narrativo canônico para a conexão consumidor-lugar

\begin{tabular}{|c|c|c|c|}
\hline (1) Manipulação & (2) Competência & (3) Performance & (4) Sanção \\
\hline $\begin{array}{l}\text { Entrevistado: "Eu tenho } \\
\text { que ir lá, porque nós } \\
\text { temos que almoçar, } \\
\text { comprar e dançar um } \\
\text { pouco. Você sabe... o } \\
\text { Nordeste é aqui." }\end{array}$ & $\begin{array}{l}\text { Entrevistado: “Eu tenho } \\
\text { certeza que a FSC é o único } \\
\text { lugar onde eu encontro os } \\
\text { produtos da minha terra } \\
\text { natal. Além disso, conheço } \\
\text { umas pessoas lá e consigo } \\
\text { algumas coisas de graça.” }\end{array}$ & $\begin{array}{l}\text { Entrevista: "Esses sons } \\
\text { estão sempre presentes } \\
\text { no mercado e têm } \\
\text { diferentes formas, } \\
\text { dependendo de onde } \\
\text { você está localizado ... } \\
\text { você pode ouvir } \\
\text { qualquer coisa, desde } \\
\text { uma apresentação da } \\
\text { Concertina até um } \\
\text { Sertanejo Universitário." }\end{array}$ & $\begin{array}{l}\text { Entrevistado: "Olha ... } \\
\text { encontrei tudo o que } \\
\text { precisava para o meu } \\
\text { almoço em família no } \\
\text { domingo. Estou dizendo a } \\
\text { você. Este é o meu lugar ... } \\
\text { estou em casa. " }\end{array}$ \\
\hline
\end{tabular}

Fonte: Elaborada pelos autores.

Durante nossas entrevistas, várias menções foram feitas sobre o papel da espacialidade em seu processo de ligação com o local. Como partes específicas do local e suas experiências vividas podem provocar emoções intensas, além de funcionar como extensões do self (HIRSCHMAN, RUVIO e BELK, 2012), investigamos onde essas experiências ocorreram na FSC. Segundo Floch (1988), o estudo das micro-narrativas dos consumidores em que os valores são destacados mostra claramente como cada tipo de avaliação pode ilustrar uma função específica do local. Portanto, a próxima seção apresenta uma análise espacial do local e seus significados para o self estendido do consumidor.

\section{Nível discursivo: análise espacial das experiências vividas na conexão consumidor-lugar}

Durante a fase final da análise, encontramos manifestações materiais de valores e significados para o self estendido do consumidor em relação às áreas de configuração comercial. Diferentemente da natureza abstrata do Nível Fundamental, o Nível Discursivo é concreto. A FSC é o lar de uma ampla variedade de atividades e experiências. Ao percorrer a FSC, o consumidor é, nos termos de Belk $(2015,1988)$ e Fernandez e Lastovicka $(2011)$, influenciado pela aura ou magia de lugares físicos, elementos materiais e pessoas. Seguindo a proposta de Floch (1988), ao examinar cada área, é possível observar e enquadrar diferentes perfis de consumidores, assim como seus valores, narrativas de consumo e apego ao ambiente comercial. A Figura 3 mostra as configurações espaciais do mercado e como ele é implantado em termos de valores atribuídos a quem o visita. 
Figura 3

\section{Mapa da FSC e as áreas com os valores e experiências de consumo}

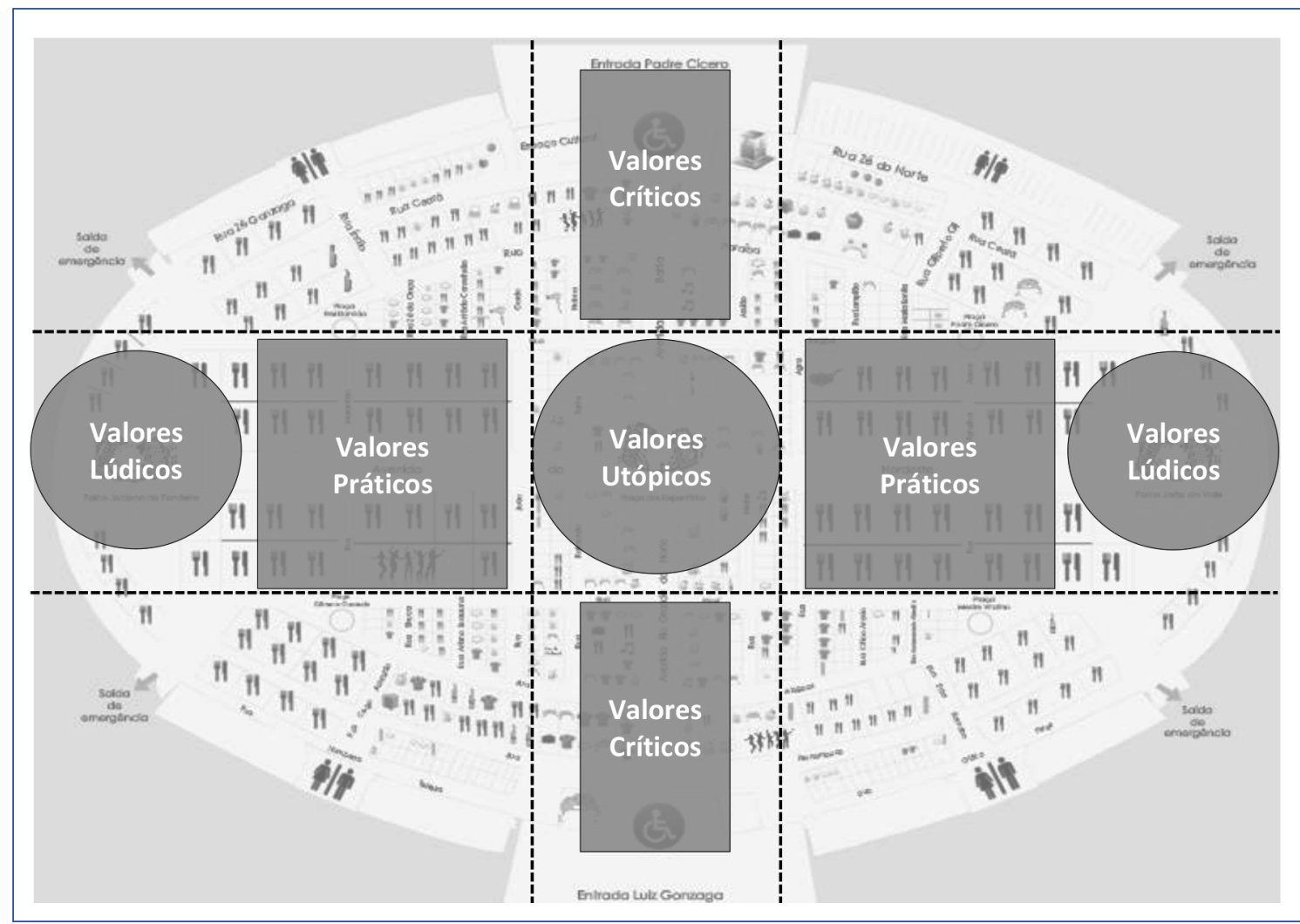

Fonte: Baseada em Feira de São Cristóvão (2018).

Duas grandes áreas, que permitem a realização de eventos ao mesmo tempo, estão localizadas nas extremidades do Pavilhão de São Cristóvão. Essas áreas têm a maior concentração de consumidores que priorizam os valores lúdicos, principalmente os hedonistas, uma vez que suas atividades no mercado estão relacionadas principalmente ao lazer e ao prazer (FLOCH, 1988), que consiste em dançar e assistir a shows. No centro do mercado fica a Praça do Repentista, onde são realizadas apresentações de cordelistas e sanfoneiros. Essa região da FSC está relacionada aos valores utópicos, onde quem prioriza as experiências de socialização entra em contato com artistas, músicas, instrumentos, objetos e outras formas de expressão cultural do Nordeste. Esses consumidores são sancionados positivamente com o contato que lhes confere a sensação de estar de volta ao Nordeste brasileiro, com suas músicas, sabores, cheiros e objetos. A rua que divide verticalmente a FSC é a que mais se alinha ao perfil consumista, ou seja, o consumidor cujo comportamento se baseia em valores críticos (FLOCH, 1998). Esta é a rua em que se encontra a maioria das lojas, lojas em geral e mercados de frutas e legumes, oferecendo produtos típicos da região, normalmente a preços inferiores aos das lojas de rua, e com uma grande variedade de ofertas. A rua que divide o mercado horizontalmente é a Avenida Nordeste, quase inteiramente dedicada a restaurantes, alinhada com os valores práticos preferência do consumidor estrategista. Além disso, os depoimentos dos entrevistados que optaram por uma experiência prática tinham uma coisa em comum, a saber, que o principal objetivo da experiência do consumidor era ir a um restaurante, em parte devido à conveniência da localização da FSC no bairro. 
A Figura 4 resume a relação entre os valores e os significados de consumo, as características da FSC que representam esses valores e os diferentes perfis de consumidores.

Figura 4

Valores relacionados com as características espaciais da FSC e o perfil dos consumidores

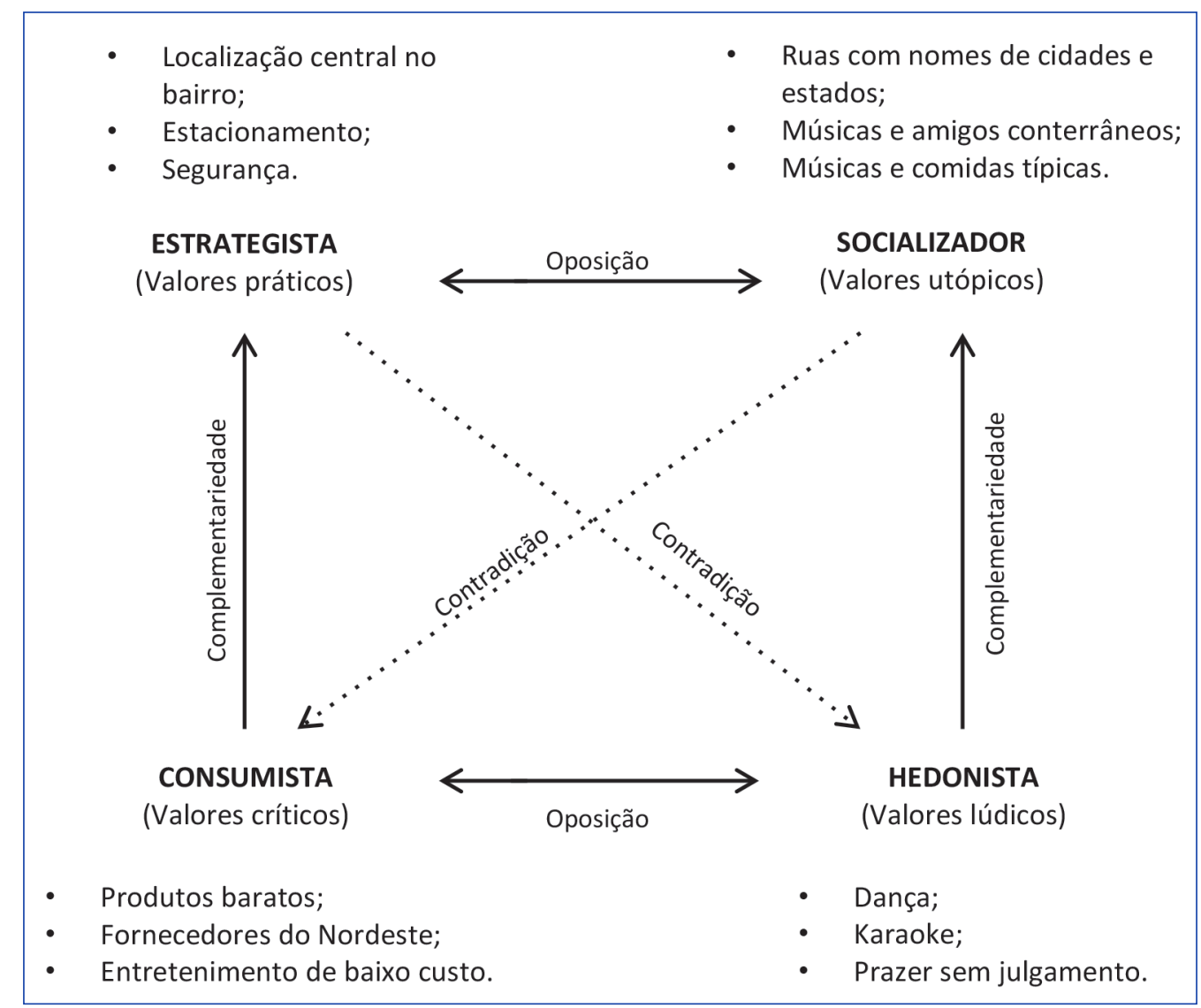

Fonte: Elaborada pelos autores.

A experiência do consumidor nas diferentes regiões da FSC possibilita diferentes significados para suas auto-narrativas, que podem ser articuladas pelo mesmo consumidor em diferentes momentos e intensidades. Tanto o aspecto simbólico quanto o espacial da FSC desempenham um papel significativo na representação de identidades. À luz disso, como todo processo de posse, a FSC tem sua própria magia (APPAU e CHURCHILL, 2019), um poder de mudar realidades e uma contribuição para o self estendido (BELK, 1988).

\section{Discussão}

Neste estudo, investigamos como os consumidores associam suas identidades a locais comerciais, baseando-se nas perspectivas da teoria do Extended Self (Belk, 1998) e do vínculo consumidor-local (e.g., SEAMON, 2018). Nossa análise se concentrou nas experiências vividas pelos visitantes nordestinos na FSC e em sua narrativa de vínculo com o local e, portanto, com sua terra natal. Mostramos como o significado é concebido a partir do contato com as propostas físicas e simbólicas da FSC e sua influência na auto-narrativa do consumidor. Os achados fornecem novas ideias sobre o processo pelo qual os consumidores vinculam sua identidade a esses ambientes comerciais, conforme solicitado por Debenedetti, Oppewal e Arsel (2014), e também para práticas gerenciais de marketing, a seguir. 


\section{Implicações teóricas}

Este estudo revela diferentes dinâmicas para o processo de vínculo entre as identidades dos consumidores e os locais comerciais. Alguns deles dependem mais de sua materialidade do que outros, mas todos eles influenciam emocionalmente a autonarrativa dos consumidores. A experiência vivida de consumir o Nordeste brasileiro na FSC, independentemente de seus significados, produtos ou do próprio local, pode ser vista como uma jornada de transcendência a partir da noção incorporada de uma determinada identidade. Ou seja, esse tipo de noção implica que o ambiente comercial tem o poder de ampliar espacialmente o senso de identidade do consumidor (BELK, 1990). Além de contribuir para a teoria do Extended Self (BELK, 1988) com uma abordagem fenomenológica, esta pesquisa também estende as discussões atuais sobre o vínculo consumidor-local. Nossos projetos de identidade, bem como suas representações, podem variar de acordo com o local em que estamos (BETTANY e BELK, 2011), mesmo quando o local é visitado para a busca de experiências dolorosas (SCOTT, CAYLA e COVA, 2017) ou por uma bênção (SANTANA e BOTELHO, 2019), ou mesmo experimentar prazer sexual (NEAL, 2018).

\section{Implicações gerenciais}

Ao observar valores e significados concebidos dentro e fora dos espaços físicos, os gerentes de marketing podem observar a Axiologia do Consumo (FLOCH, 1988) para elaborar melhores jornadas de consumo. Conforme observado por Payne, Peltier e Barger (2017), um entendimento adequado da jornada de compra é fundamental para estimular o comportamento desejado e resultados financeiros. Além disso, todas as práticas de consumo no local geram diferentes tipos de dados, que podem ser rastreados e utilizados para fins de análise digital (FULGONI, 2018). Outra implicação prática diz respeito a campanhas publicitárias. As experiências vividas na FSC e os significados resultantes para o self podem ser explorados em anúncios, tanto offline quanto online. Em tempos de profunda conexão e, às vezes, de dependência do mundo digital (BELK, 2013), projetar um plano eficaz de comunicação de marketing integrado se apresenta como um desafio. Dada a nossa abordagem semiótica, um gerente que esteja interessado em melhorar a comunicação com seus consumidores poderá considerar esta pesquisa para entender a articulação de significados enunciados, expectativas e percepções sobre narrativas de consumo que poderiam ser retratadas na publicidade. Além disso, os gerentes também podem concentrar seus esforços na construção de um maior senso de comunidade de marca, já que a conexão entre o consumidor nordestino e o ambiente comercial é altamente significativa.

\section{Limitações e oportunidades}

Como todas as pesquisas, este estudo qualitativo possui limitações que exigem atenção. Como o contexto da pesquisa se refere a um mercado regional e, devido a história da FSC e a sua relevância para as identidades dos entrevistados, não deve ser comparado a um shopping center ou a um hipermercado localizado no nordeste brasileiro, pelo menos no início. Ou seja, a FSC é um simulacro de uma realidade brasileira específica, que pode levar a uma oportunidade de pesquisa de contextos distintos, como feiras, mercados itinerantes, outras cidades etc. (e.g., BARROSO, PESSÔA e LIMA, 2020). Quanto à abordagem metodológica, as observações, entrevistas em profundidade (BELK, FISCHER e KOZINETS, 2013) e análise de discurso (GREIMAS e COURTÉS, 1983) são limitadas. Em vez de focar na generalização, nosso esforço concentrou-se em saber se as descobertas e ideias do estudo poderiam ser transportadas adequadamente para outros contextos (MILES e HUBERMAN, 2014). Embora os estudos de consumo tenham a fenomenologia existencial como um de seus principais paradigmas, o uso de uma abordagem teórico-metodológica diferente (ASKEGAARD e LINNET, 2011) é sugerido para futuros estudos sobre a dinâmica de forças macro-sociais que podem influenciar a conexão consumidor-lugar. Outra sugestão é que pesquisadores possam explorar a proposta de Floch (1988) considerando uma audiência mais ampla, dada a especificidade dos perfis dos participantes. 


\section{REFERÊNCIAS}

ABDALLA, C.; ZAMBALDI, F. Ostentation and funk: An integrative model of extended and expanded self theories under the lenses of compensatory consumption. International Business Review, v. 25, n. 3, p. 633-645, 2016.

AHUVIA, A. Beyond the extended self: Loved objects and consumers' identity narratives. Journal of Consumer Research, v. 32, n. 1, p. 171-184, 2005.

ALTMAN, I.; LOW, S. Place Attachment. Boston: Springer, 1992.

APPAU, S.; CHURCHILL, S. Bridging cultural categories of consumption through indeterminacy: A consumer culture perspective on the rise of African Pentecostal-Charismatic Christianity. Journal of Consumer Culture, v. 19, n. 1, p. 125-145, 2019.

ASKEGAARD, S.; LINNET, J. Towards an epistemology of consumer culture theory: Phenomenology and the context of context. Marketing Theory, v. 11, n. 4, p. 381-404, 2011.

BARROSO, F.; PESSÔA, L.; LIMA, V. A construção identitária da marca Rio sob a perspectiva. Diálogo com a Economia Criativa, v. 5, n. 13, p. 142-159, 2020.

BELK, R. Ownership: The extended self and the extended object. In: Peck, J.; Shu, S. (ed.) Psychological ownership and consumer behavior. New York: Springer, 2018.

BELK, R. Object Agency and the Extended Object. In: Wan, E.; Zhang, M. (ed.). AP - Asia-Pacific Advances in Consumer Research. Duluth: Association for Consumer Research. v.11, 2015.

BELK, R. Extended self in a digital world. Journal of Consumer Research, v. 40, n. 3, p. 477-500, 2013.

BELK, R.; FISCHER, E.; KOZINETS, R. Approaches to data analysis, interpretation and theory building for scholarly research. Qualitative Consumer and Marketing Research. London: Sage, 2013.

BELK, R. Attachment to possessions. In: Altman, I.; Low, S. (ed.). Place Attachment. Boston: Springer, 1992.

BELK, R. Possessions and the Extended Self. Journal of Consumer Research, v. 15, n. 2, p. 139-168, 1988.

BETTANY, S.; BELK, R. Disney discourses of self and other: Animality, primitivity, modernity, and postmodernity. Consumption Markets and Culture, v.14, n. 2, p. 163-176, 2011.

BORRAZ, S. Love and Locks: Consumers Making Pilgrimages and Performing Love Rituals, In: BAJDE, D.; KJELDGAARD, D.; BELK, R. (ed.). Consumer Culture Theory (Research in Consumer Behavior. London: Emerald Publishing Limited, 2019.

BRAGA, C.; SUAREZ, M. Actor-network theory: new perspectives and contributions to consumption studies. Cadernos EBAPE.BR, Rio de Janeiro, v. 16, n. 2, p. 218-231, 2018.

CANNIFORD, R.; RIACH, K.; HILL, T. Nosenography: How smell constitutes meaning, identity and temporal experience in spatial assemblages. Marketing Theory, v.18, n.2, p. 234-248, 2018.

COVA, B.; CARÙ, A.; CAYLA, J. Re-conceptualizing escape in consumer research. Qualitative Market Research: An International Journal, v.21, n.4, p. 445-464, 2018.
CASEY, E. Remembering: A phenomenological study. Bloomington: Indiana University Press, 2009.

CHATZIDAKIS, A.; MCEACHERN, M.; WARNABY, G. Virtual special issue: The concept of place in marketing theory and research. Marketing Theory, 2014. Available at: <https://journals.sagepub.com/pb-assets/ cmscontent/MTQ/MTQ-Concept-of-Place-Intro-1471959826993. pdf>. Acesso em: 01 nov. 2019.

DEBENEDETTI, A.; OPPEWAL, H.; ARSEL, Z. Place attachment in commercial settings: A gift economy perspective. Journal of Consumer Research, v. 40, n.5, p. 904-923, 2014.

EROGLU, S.; MICHEL, G. The dark side of place attachment: Why do customers avoid their treasured stores? Journal of Business Research, v. 85, p. 258-270, 2018.

FEIRA DE SÃO CRISTÓVÃO. "Feira de São Cristóvão". Disponível em: <https://www.feiradesaocristovao.org.br>._Acesso em: 29 dez. 2018.

FERNANDEZ, K.; LASTOVICKA, J. Making magic: Fetishes in contemporary consumption. Journal of Consumer Research, v. 38, n.2, p. 278299, 2011.

FERREIRA, M.; SCARABOTO, D. "My plastic dreams": Towards an extended understanding of materiality and the shaping of consumer identities. Journal of Business Research, v. 69, n.1, p. 191-207, 2016.

FLOCH, J. The contribution of structural semiotics to the design of a hypermarket. International Journal of Research in Marketing, v.4, n.3, p. 233-252, 1988.

FULGONI, G. Will Digital Commerce and Analytics Be the Death of Traditional Brands?. Journal of Advertising Research, v.58, n.2, p. 146-150, 2018.

GAVIRIA, P.; CARDOSO, F., SCARABOTO, D., \& DE ARAUJO GIL, L. Motherhood in migration: schools as acculturation agents. Consumption Markets and Culture, v.22, n.4, p. 1-23, 2019.

GIOVANARDI, M.; LUCARELLI, A. Sailing through marketing: A critical assessment of spatiality in marketing literature. Journal of Business Research, v. 82, n. 1, p. 149-159, 2018.

GOWRICHARN, R. Practices in taste maintenance. The case of Indian diaspora markets. Journal of Consumer Culture, v. 19, n. 3, p. 398416, 2019.

GREIMAS, A.; COURTÉS, J. Semiotics and Language: An Analytical Dictionary. Bloomington: IU Press, 1983.

GUILLARD, V. Understanding the process of the disposition of a loved one's possessions using a theoretical framework of grief. Consumption Markets and Culture, v. 20, n. 5, p. 477-496, 2017.

JAMES, W. The Principles of Psychology. New York: Henry Holt, 1890.

HIRSCHMAN, E.; RUVIO, A.; BELK, R. Exploring space and place in marketing research: Excavating the garage. Marketing Theory, v. 12, n. 4, p. 369-389, 2012.

HOLBROOK, M. Stereo 3D representations in postmodern marketing research. Marketing Intelligence \& Planning, v. 16, n. 5, p. 298310, 1998 
HOLBROOK, M.; HIRSCHMAN, E. The experiential aspects of consumption: Consumer fantasies, feelings, and fun. Journal of Consumer Research, v. 9, n. 2, p. 132-140, 1982.

ISABELLE, F.; DOMINIQUE, K.; STATIA, E. Home away from home: A longitudinal study of the holiday appropriation process. Tourism Management, v. 71, n. 04, p. 327-336, 2019.

JÚNIOR, E.; PORTO, E. Feira de São Cristovão: Patrimônio Cultural, Histórico e Artístico. Caderno Virtual de Turismo, v. 2, n. 3, p. 11-16, 2002.

KOZINETS, R. Netnography: The Essential Guide to Qualitative Socia Media Research. London, UK: SAGE Publications Ltd, 2019.

KOZINETS, R. V.; PATTERSON, A.; ASHMAN, R. Networks of desire: How technology increases our passion to consume. Journal of Consumer Research, v. 43, n. 5, p. 659-682, 2017.

KREUZER, M.; MÜHLBACHER, H.; VON WALLPACH, S. Home in the re-making: Immigrants' transcultural experiencing of home. Journal of Business Research, v. 91, n. 10, p. 334-341, 2018.

LIMA, V.; PESSÔA, L. The consumption of technology as a structural element of identity: a theoretical and methodological discussion based on French Semiotics. Cadernos EBAPE.BR, Rio de Janeiro, v. 16, n. 4, p. 679-691, 2018.

LIMA, V. et al. Branding Rio de Janeiro: a semiotic analysis. Marketing Intelligence \& Planning, v. 37, n. 6, p. 645-659, 2019.

LOW, S. Symbolic Ties That Bind: Place Attachment in the Plaza. In: ALTMAN, I.; LOW, S. (ed.). Place Attachment. Boston: Springer, 1992.

MANCEBO, R. Mídia, discurso e operação ideológica. Disponível em: <www2.dbd.puc-rio.br\%2Fpergamum\%2 Ftesesabertas\%2F1412502_2016_completo.df\&usg=AOvVaw07sPXk-z ioY_MV37MQZ9Y>. Acesso em: 10 out. 10, 2019.

MARCOUX, J. Souvenirs to forget. Journal of Consumer Research, v. 43, n. 6, p. 950-969. 2017.

MCCRACKEN, G. "Homeyness": a cultural account of one constellation of consumer goods and meanings. In: E. Hirschman (ed.). SV Interpretive Consumer Research. Provo: Association for Consumer Research, 1989.

MEHTA, R.; BELK, R. Artifacts, identity, and transition: Favorite possessions of Indians and Indian immigrants to the United States. Journal of Consumer Research, v.17, n. 4, p. 398-411, 1991.

MILES, M.; HUBERMAN, A. Qualitative data analysis: a methods sourcebook. London: Sage, 2014.

NEAL, M. Dirty customers: Stigma and identity among sex tourists. Journal of Consumer Culture, v.18, n. 1, p. 131-148, 2018.

PAYNE, E.; PELTIER, J.; BARGER, V. Omni-channel marketing, integrated marketing communications and consumer engagement: $A$ research agenda. Journal of Research in Interactive Marketing, v. 11, n. 2, p. 185-197, 2017.
PESSÔA, L. et al. Nostalgia in Brazilian Mass Media Advertising: A Semiotic Perspective. Latin American Business Review, 1-28, in-press, 2020.

PULH, M.; MENCARELLI, R.; CHANEY, D. The consequences of the heritage experience in brand museums on the consumer-brand relationship. European Journal of Marketing, v. 53, n. 10, p. 2193 2212, 2019.

RISHBETH, C.; POWELL, M. Place attachment and memory: Landscapes of belonging as experienced post-migration. Landscape Research, v. 38, n. 2, p. 160-178, 2013.

ROSENBERG, B. (2012). Dangerous Houses: Scientific Lifestyle Television and Risk Management. Home Cultures, v. 9, n. 2, p. 173-194, 2012.

RUVIO, A.; BELK, R. Strategies of the extended self: The role of possessions in transpeople's conflicted selves. Journal of Business Research, v. 88, n. 7, p. 102-110, 2018.

SANTANA, J.; BOTELHO, D. 'If it comes from Juazeiro, it's blessed'! Liquid and solid attachment in systems of object itineraries of pilgrimages. Journal of Marketing Management, v. 35, n. 6, p. 514$539,2019$.

SCHAU, H. Identity Projects and the Marketplace. In: ARNOULD, E.; THOMPSON, C. (ed.). Consumer Culture Theory. London: Sage Publications, 2018.

SCOTT, R.; CAYLA, J.; COVA, B. Selling pain to the saturated self Journal of Consumer Research, v. 44, n. 1, p. 22-43, 2017.

SEAMON, D. Life takes place: Phenomenology, lifeworlds, and place making. London: Routledge, 2018.

TERZIDOU, M.; SCARLES, C.; SAUNDERS, M. Religiousness as tourist performances: A case study of Greek Orthodox pilgrimage. Annals of Tourism Research, v. 66, n. 9, p. 116-129, 2017.

THOMPSON, C.; TAMBYAH, S. Trying to be cosmopolitan. Journal of Consumer Research, v. 26, n. 3, p. 214-241, 1999.

TIAN, K.; BELK, R. Extended self and possessions in the workplace. Journal of Consumer Research, v. 32, n. 2, p. 297-310, 2005.

TSAI, C. Memorable tourist experiences and place attachment when consuming local food. International Journal of Tourism Research, v. 18, n. 6, p. 536-548, 2016.

VERESIU, E.; GIESLER, M. Beyond acculturation: Multiculturalism and the institutional shaping of an ethnic consumer subject. Journal of Consumer Research, v.45, n. 3, p. 553-570, 2018.

WALLENDORF, M.; BELK, R. Assessing Trustworthiness in Naturalistic Consumer Research. In: HIRSCHMAN, E. (ed.). SV - Interpretive Consumer Research. Provo: Association for Consumer Research, 1989.

ZANETTE, M.; LOURENÇO, C.; BRITO, E. The weight of retail, the weight in retail and identity: an analysis of plus size consumers. Revista de Administração de Empresas, v. 53, n. 6, p. 539-550, 2013. 
Vitor Moura Lima

ORCID: https://orcid.org/0000-0001-6750-1641

Postdoctoral Fellow na Schulich School of Business, York University, Toronto - ON, Canadá. E-mail: Imv2607@yorku.ca

Rafael Cuba Mancebo

ORCID: https://orcid.org/0000-0001-6297-2399

Professor visitante no IAG Escola de Negócios- Pontifícia Universidade Católica do Rio de Janeiro (PUC-Rio), Rio de Janeiro- RJ, Brasil.

E-mail: cuba.mancebo@gmail.com

Luís Alexandre Grubits de Paula Pessôa

ORCID: https://orcid.org/0000-0002-1532-6084

Professor no IAG Escola de Negócios- Pontifícia Universidade Católica do Rio de Janeiro (PUC-Rio), Rio de Janeiro- RJ, Brasil.

E-mail: Ipessoa@iag.puc-rio.br

Alessandra de Sá Mello da Costa

ORCID: http://orcid.org/0000-0003-3207-2888

Professora no IAG Escola de Negócios- Pontifícia Universidade Católica do Rio de Janeiro (PUC-Rio), Rio de Janeiro- RJ, Brasil.

E-mail: alessandra.costa@iag.puc-rio.br 